\title{
SMART GRID FOR OPTIMAL ENERGY MANAGEMENT USING RENEWABLE ENERGY
}

Ananthan Nagarajan

Assistant Professor

Department of EEE

Vel Tech (Owned by RS Trust), Chennai, India,

\author{
A. Anugraha, \\ Assistant Professor, \\ Department of EEE, \\ Vel Tech (Owned by RS \\ Trust), Chennai, India
}

\author{
N. Dhanalakshmi \\ U.G.Students, \\ Department of EEE, \\ Vel Tech (Owned by RS \\ Trust), Chennai, India
}

\author{
T. Divyasheeba \\ UG Students, \\ Department of EEE, \\ Vel Tech (Owned by RS \\ Trust), Chennai, India
}

\author{
S. Nivetha \\ UG Students, \\ Department of EEE, \\ Vel Tech (Owned by RS \\ Trust), Chennai, India
}

\begin{abstract}
Need for energy is increasing considerably with increase in power demand around he world, that burdens standard grid (CG) because of peak demand. Majority of the countries have plenty of wind and solar energy which can be harnessed locally for optimal energy generation. Major objective of this project is to reduce the peak power deficit present in CG system during peak demand and to provide a reliable power supply even in case of grid failure or during blackout. In this project, development and evaluation of a small scale grid interactive DC micro-grid (DCMG) for residential houses, telecommunication systems and data centre has been proposed. The objective is to achieve by a comprehensive power flow control strategy through real time simulation in RSCAD/RTDS platform. A Prototype is developed to validate the simulation results present during grid connection and isolation mode
\end{abstract}

Kewords-. Conventional Grid, Energy Management System, PIC Microcontroller, Solar Panel, Two relay board.

\section{INTRODUCTION}

An energy management system \&\#40;EMS\&\#41; allows for centralized monitoring and control of energy use across building systems. The upgrades to controls for lighting, workplace instrumentation, HVAC, and water heating represent for "stand-alone" management systems (e.g., photo sensor-based dimming controls for lighting) (Lee et al.,2016; Lim 2015; Ker et al.,2011; Jo et al.,2012; Thotahewa et al.,2013). An EMS is a "central" control system, allowing facility managers to operate all stand-alone control systems in a building simultaneously from a single control pad or web application. Sensors throughout the building that live conditions like light-weight level, indoor/outdoor temperature, and water temperature (called "monitoring points") function knowledge inputs for the EMS, which uses that information to regulate management parts (called "control points") like dimmers, chillers, and boilers. are When a new EMS is installed, it can be configured to work with most existing sensors and controls, and to any new monitoring points and control points that added. In recent years, EMS technologies have become more affordable and more widely used ( Che et al.,2015; Nejabatkhah, and Li 2015; Xiao et al.,2015; Wu et al.,2015; Nordman et al.,2012)

\section{SOLAR PANEL}

, but rather converted into the final published version.

Solar panel refers to a panel designed to soak up the sun's rays as a supply of energy for generating electricity or heating. star panels represent the electrical device of a electrical phenomenon system that generates and provides star electricity in industrial and residential applications. Each module is rated by its DC output power below commonplace check conditions. Solar modules use light Efficiencies of solar panel can be calculated by MPP (Maximum power point) value of solar panels. It is a capability of the solar battery and therefore the higher price will create higher MPP. (Kollimalla et al.,2014; Kinhekar et al.,2016; Grillo et al.,2014; Indu Rani et al.,2013). These panels square measure designed for the foremost rugged off-grid applications. A electrical phenomenon system generally includes a panel or associate degree array of star modules, a solar inverter, and sometimes a battery and/or solar tracker and interconnection wiring. A single star module will turn out solely a restricted quantity of power, most installations contain multiple modules. The majority of modules use wafer-based crystalline chemical element cells or thin-film cells supported metal compound or chemical element. Cells should even be protected against mechanical harm and wetness. Most star modules square measure rigid, but semi-flexible ones are available, based on thin film cells. Electrical connections square measure created 


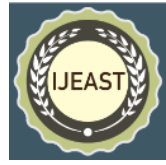

asynchronous to realize a desired output voltage and/or in parallel to produce a desired current (Eghtedarpour. and Farjah2014; Majumder 2014)

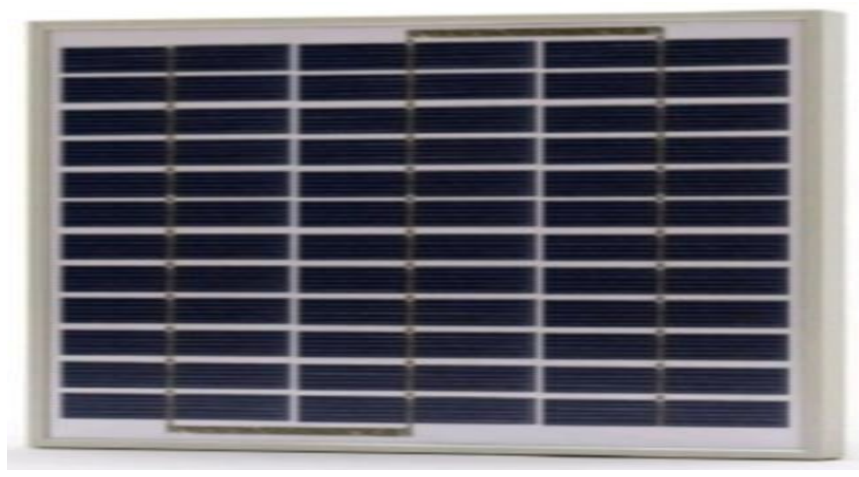

Fig. 1. Solar Panel

\section{PIC MicrocontrolleR}

PIC16F877A: The 16F877A may be a capable microcontroller which will do several tasks as a result of it's an oversized enough programming memory (large in terms of device and management projects) $8 \mathrm{k}$ words and 368 Bytes of RAM. This is enough to do many different projects. The forty pins create it easier to use the peripherals because the functions square measure displayed over the pins. This makes it easier to choose what external devices to connect without fear an excessive amount of if there square measure enough pins to try and do the work. One of the most blessings is that every pin is merely shared between 2 or 3 performs thus its easier to choose what the pin function.

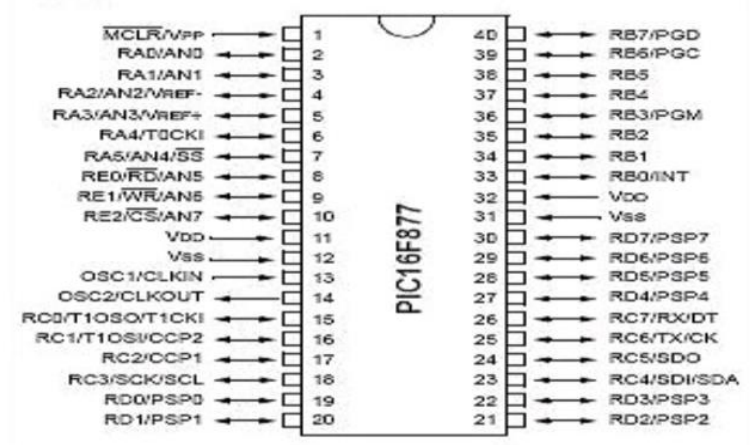

Fig. 2. Pin Diagram Of PIC16F877

This powerful (200 time unit instruction execution) however easy-to-program (only thirty five single word instructions) CMOS FLASH-based 8-bit microcontroller packs Microchip's powerful PIC design into associate degree 40- or 44-pin package and is upwards compatible with the PIC16C5X, PIC12CXXX and PIC16C7X devices. The PIC16F877A options 256 bytes of EEPROM knowledge memory, self programming, an ICD, 2 Comparators, 8 channels of 1Analog-to-Digital(A/D) converter, 2 capture/compare/PWM functions, the synchronous serial port. Transmitter (USART). All of those options create it ideal for additional advanced level.

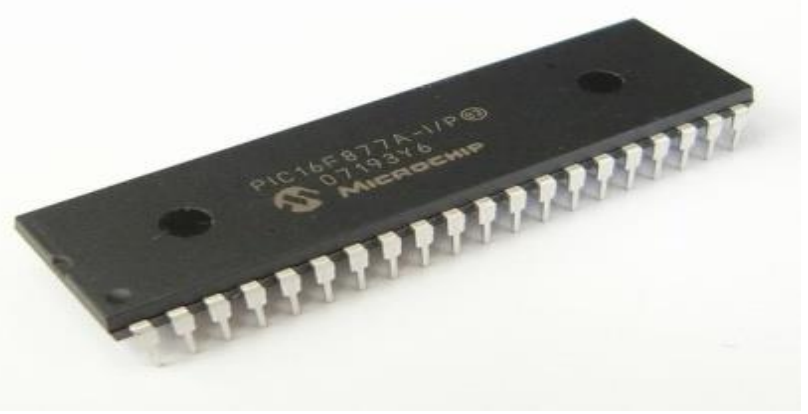

Fig. 3. PIC16F877A

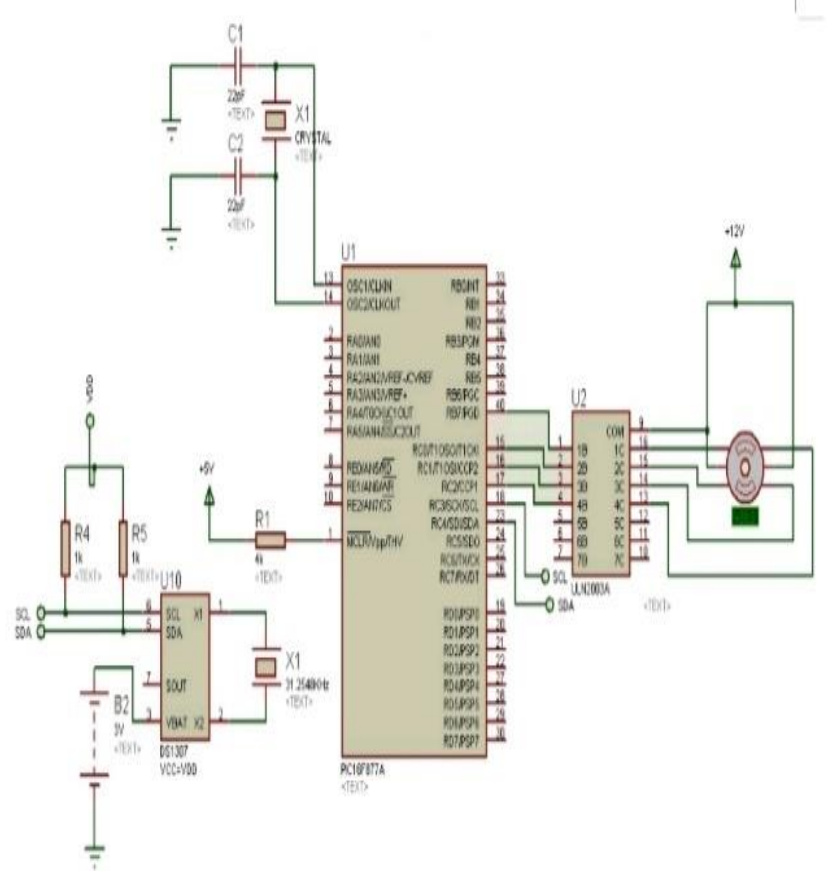

Fig. 4. Circuit Diagram Of PIC16F877A

\section{Two RELAY BOARD}

Relays square measure straightforward switches that square measure operated each electrically and automatically. Relays comprises a $\mathrm{n}$ magnet and additionally a collection of contacts. The switch mechanism is dole out with the assistance of the magnet. The main operation of a relay comes in places wherever solely a low-power signal are often accustomed management a circuit. It is additionally utilized in places wherever only 1 signal are often accustomed management a great deal of circuits. 
They were accustomed switch the signal coming back from one supply to a different destination. The high finish applications of relays need high power to be driven by electrical motors then on. Such relays are called contactors.

A relay is associate degree mechanical device switch that is activated by an electrical current. A four relay board arrangement contains driver circuit, power supply circuit and isolation circuit. A relay is assembled with that circuit. The driver circuit contains transistors for switching operations. The transistor is use for switching the relay.

An isolation circuit prevents reverse voltage from the relay that protects the controller and semiconductor device from harm. The input pulse for switch the semiconductor device is given from the microcontroller unit. It is used for switch of a 2 device.

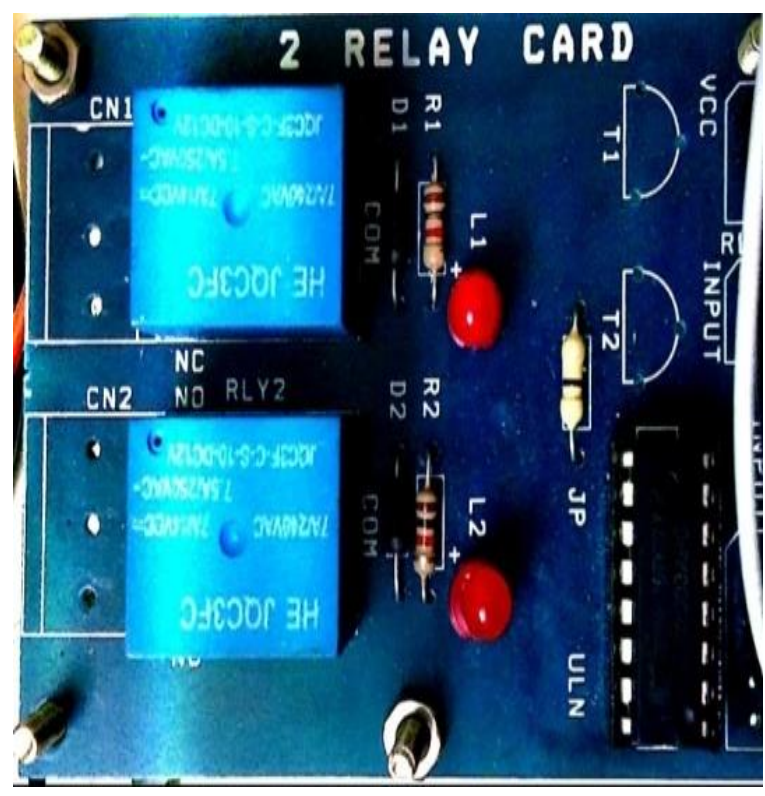

Fig. 5. Two Relay Board

\section{WORKING PRINCIPLE}

In this system we are using solar energy as a renewable source were the heat energy is converted into electrical energy.

The power obtained from it is given to the smart switching network through the bus bar the excess power it fed to the battery for the future use.

The smart switch in network gives the power to both the energy backup system and to the programmable circuits where the energy backup system gives the display of power usage by the use of utility grid and the relay circuits are used for the switching according to the demand.

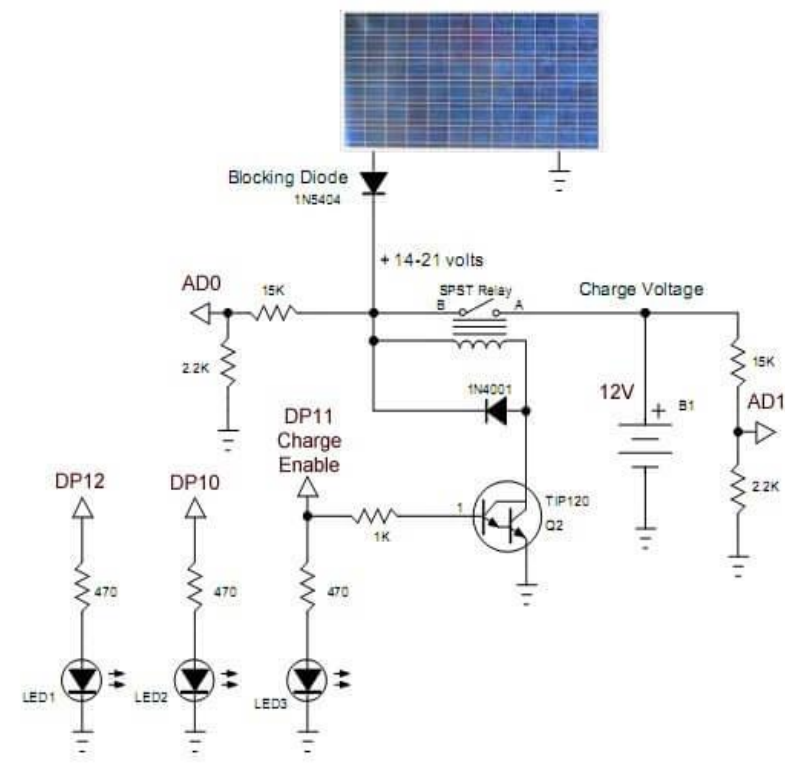

Fig. 6. Proposed System Circuit

VI. SOURCE CODE

\section{SOURCE CODE FOR ENERGY AUDITING}

clc;

clear all;

close all;

warning off;

load simpleclass_dataset;

$\mathrm{x}=$ simpleclassInputs;

$\mathrm{t}=$ simpleclassTargets;

trainFen = 'trainscg'; \% Scaled conjugate gradient backpropagation.

\% Create a Pattern Recognition Network

hiddenLayerSize $=10$;

net $=$ patternnet(hiddenLayerSize, trainFen);

$\%$ Choose Input and Output Pre/Post-Processing Functions

$\%$ For a list of all processing functions type: help nnprocess net.input.processFens

\{'removeconstantrows','mapminmax'\};

\% Setup Division of Data for Training, Validation, Testing

$\%$ For a list of all data division functions type: help nndivision net.divideFcn = 'dividerand'; \% Divide data randomly net.divideMode = 'sample'; \% Divide up every sample net.divideParam.trainRatio $=70 / 100$;

net.divideParam.valRatio $=15 / 100$;

net.divideParam.testRatio $=15 / 100$;

$\%$ Choose a Performance Function

$\%$ For a list of all performance functions type: help nnperformance

net.performFen = 'crossentropy'; \% Cross-Entropy

$\%$ Choose Plot Functions 
$\%$ For a list of all plot functions type: help nnplot net.plotFcns $=\{$ 'plotperform','plottrainstate','ploterrhist', ... 'plotconfusion', 'plotroc'\};

$\%$ Train the Network

$[$ net, $t r]=$ train $($ net $, \mathrm{x}, \mathrm{t})$;

$\%$ Test the Network

$\mathrm{y}=\operatorname{net}(\mathrm{x})$;

$\mathrm{e}=\operatorname{gsubtract}(\mathrm{t}, \mathrm{y})$

performance $=$ perform $($ net, $t, y)$

tind $=\operatorname{vec} 2 \operatorname{ind}(\mathrm{t})$;

yind $=\operatorname{vec} 2$ ind $(y)$;

percentErrors $=$ sum(tind $\sim=$ yind $) /$ numel(tind $)$;

$\%$ Recalculate Training, Validation and Test Performance

trainTargets $=\mathrm{t} . *$ tr.trainMask $\{1\}$;

valTargets $=\mathrm{t} . * \operatorname{tr} . \operatorname{valMask}\{1\}$;

testTargets $=\mathrm{t} . *$ tr.testMask $\{1\}$;

trainPerformance $=$ perform $($ net, trainTargets, $y)$

valPerformance $=$ perform $($ net, valTargets, $y$ )

testPerformance $=$ perform $($ net, testTargets,y)

$\%$ View the Network

view(net)

$\%$ Plots

$\%$ Uncomment these lines to enable various plots.

figure, plotperform(tr)

figure, plottrainstate(tr)

figure, ploterrhist(e)

\%figure, plotconfusion $(\mathrm{t}, \mathrm{y})$

figure, plotroc(t,y)

$\%$ Deployment

$\%$ Change the (false) values to (true) to enable the following code blocks.

$\%$ See the help for each generation function for more information.

if (false)

$\%$ Generate MATLAB function for neural network for application

$\%$ deployment in MATLAB scripts or with MATLAB Compiler and Builder

$\%$ tools, or simply to examine the calculations your trained neural

$\%$ network performs.

genFunction(net,'myNeuralNetworkFunction'); end

$\mathrm{y}=$ myNeuralNetworkFunction( $\mathrm{x}$ );

if (false)

$\%$ Generate a matrix-only MATLAB function for neural network code

$\%$ generation with MATLAB Coder tools.

genFunction(net,'myNeuralNetworkFunction','MatrixOnly' ,'yes')

$\mathrm{y}=$ myNeuralNetworkFunction(x); end

if (false)

$\%$ Generate a Simulink diagram for simulation or deployment with.
$\%$ Simulink Coder tools. gensim(net); end

\section{ADVANTAGE}

- More number of sources are used

- The display unit determines the amount of power

- Advanced algorithm is used

\section{APPLICATIONS}

- House hold appliances

- Office equipment

- Instruments

- Peripheral devices

- Motor control

\section{RESUlT}

In our project, the main aim is to design an Energy Management system using grid interactive DC micro grid. The major outcome of this project is to reduce the peak power deficit present in $\mathrm{CG}$ system during peak demand and to provide a reliable power supply even in case of grid failure or during black out.

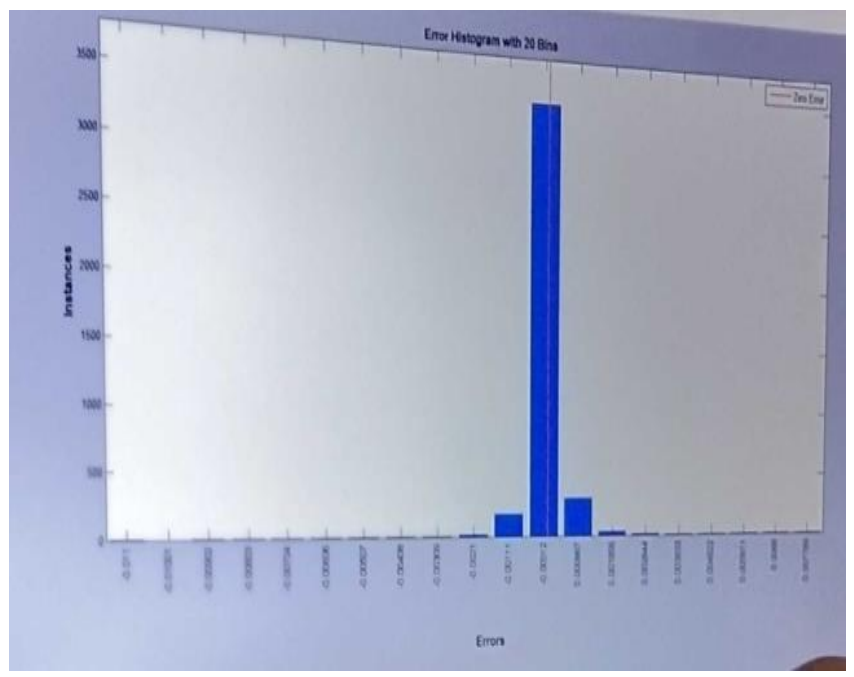

Fig. 7. Simulation Output for Error Histogram

Another outcome of this project is to achieve a comprehensive power flow control strategy through RTDS/RSCAD platform. A prototype is developed to validate the simulation result present during grid connection and isolation mode.

Thus, the Energy Auditing is done to determine the voltage and current consumed by the load. 


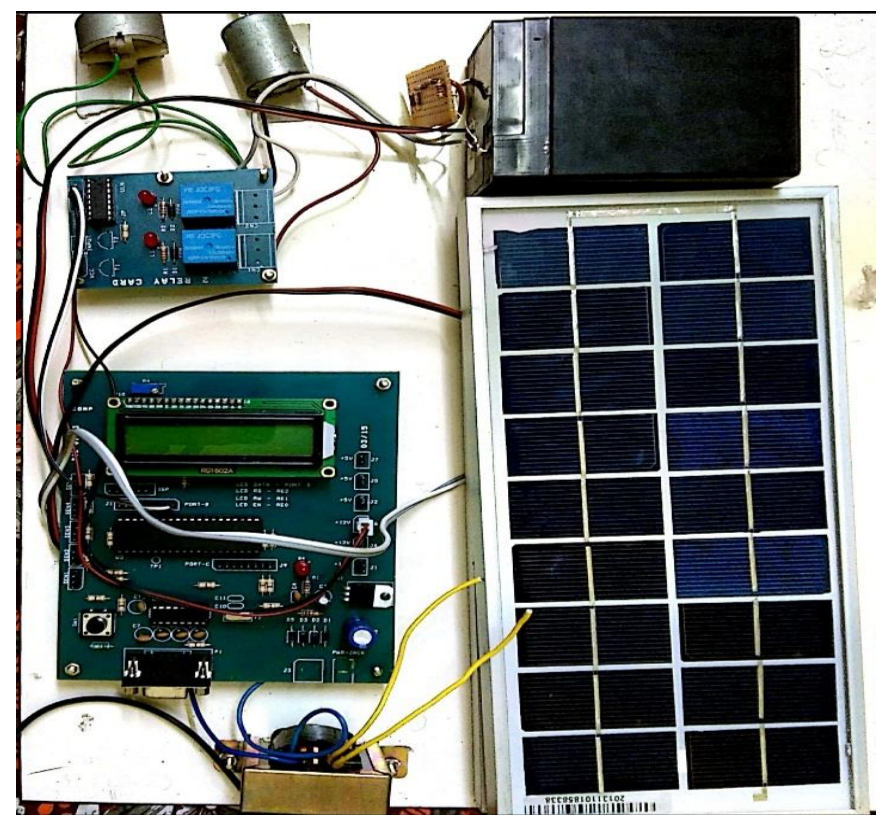

Fig. 8. Hardware

\section{CONCLUSION}

A simplified power flow management scheme is proposed for minimizing the peak demand as well as ensuring optimal utilization of renewable energy and storage. The performance of the developed strategy is exposed under various practical conditions to ensure the reliable and efficient operation. The simulation results were satisfactory and the micro grid remained even in case of extreme conditions. Peak demand detection, diminishing of stress on CG network, pervasive control analysis are observed as main ass features of proposed scheme. The results also emphasized on the independence from centralized grid thus increasing reliability in case of grid outages. The DCMG is found to be suitable for large applications and even remote villages where CG do not exist or unreliable.

\section{REFERENCE}

[1] Lee, B.,Kiani, M. and Ghovanloo,M.(2016). A TripleLoop Inductive Power Transmission System for Biomedical Applications IEEE Transactions on Biomedical Circuits and Systems, 10(1),138-148,

[2] Lim,H., et al.,(2015) Wireless charing pillow for a fully implantable hearing aid: Design of a circular array coil based on finite element analysis for reducing magnetic weak zones Bio-Medical Materials and Engineering, 26(1),S1741-S1747,

[3] Ker,M.,Lin,C and Chen,W.(2011)Stimulus driver for epilepsy seizure suppression with adaptive loading impedance Journal of Neural Engineering, 8(6) 066008,
[4] Jo,S, Joung,,S., Suh,J and Kim,Y.(2012) Improvement of wireless power transmission efficiency of implantable subcutaneous devices by closed magnetic circuit mechanism Medical \& Biological Engineering \& Computing, 50(9) 973-980,

[5] Thotahewa,K.,,Redoute,J. and Yuce,M.(2013) Electromagnetic and thermal effects of IR-UWB wireless implant systems on the human head 2013 35th Annual International Conference of the IEEE Engineering in Medicine and Biology Society (EMBC),

[6] Che,L..Shahidehpour,m., Alabdulwahab,A. and AlTurki,Y.(2015) Hierarchical Coordination of a Community Microgrid With AC and DC Microgrids IEEE Transactions on Smart Grid, 6(6),3042-3051,

[7] Nejabatkhah,F., and Li,Y.(2015) Overview of Power Management Strategies of Hybrid AC/DC Microgrid IEEE Transactions on Power Electronics,. 30(12), 7072-7089

[8] Xiao,J., Wang,P. and Setyawan,L(2015)Multilevel Energy Management System for Hybridization of Energy Storages in DC Microgrids IEEE Transactions on Smart Grid,. 1-1,

[9] Wu,D., Tang,F., Dragicevic,T., Guerrero,J. and Vasquez,J.(2015) Coordinated Control Based on BusSignaling and Virtual Inertia for Islanded DC Microgrid, IEEE Transactions on Smart Grid, 6(6,) 2627-2638,

[10] Nordman,B., Christensen,K. and Meier,A.(2012)Think Globally, Distribute Power Locally: The Promise of Nanogrids Computer, 45(9),.89-91,

[11] Kollimalla,S., Mishra,M. and Narasamma,N.,(2014) Design and Analysis of Novel Control Strategy for Battery and Supercapacitor Storage System IEEE Transactions on Sustainable Energy, 5, (4) 1137-1144,

[12] Kinhekar,N.,Padhy,N., Li,F. and Gupta,H(2016)Utility Oriented Demand Side Management Using Smart AC and Micro DC Grid Cooperative IEEE Transactions on Power Systems, 31,(2),1151-1160,

[13] Grillo,S., Musolino,V., Piegari,L., Tironi ,E.and Tornelli,C(2014).DC Islands in AC Smart Grids IEEE Transactions on Power Electronics, 29(1),. 89-98,

[14] Indu Rani,B., Saravana Ilango,G. and Nagamani, C(2013)Control Strategy for Power Flow Management in a PV System Supplying DC Loads IEEE Transactions on Industrial Electronics, 60.(8), 31853194 ,

[15].Eghtedarpour.N. and Farjah,E.(2014). Power Control and Management in a Hybrid AC/DC Microgrid, IEEE Transactions on Smart Grid,. 5.(3),. 1494-1505.

[16]Majumder,R.,(2014)A Hybrid Microgrid With DC Connection at Back to Back Converters IEEE Transactions on Smart Grid, . 5,(1), 251-259, 